\title{
A new intense DC muon beam from a pion capture solenoid, MuSIC
}

\author{
Y. Hino' ${ }^{1}$ I. H. Hashim, Y. Kuno, A. Sato, H. Sakamoto, N. H. Tran, M. Truong \\ Department of Physics, Osaka University \\ 1-1 Machikaneyama, Toyonaka, Osaka 560-0043, Japan
}

K. Hatanaka, M. Fukuda

Research Center of Nuclear Physics (RCNP), Osaka University

T. Ogitsu, A. Yamamoto, M. Yoshida

High Energy Accelerator Research Organization (KEK)

\section{Y. Mori}

Kyoto University Research Reactor Institute

\section{S. Cook, A. Edmonds, M. Lancaster, M. Wing}

Department of Physics, university of College London

\begin{abstract}
MuSIC is a project to provide the world's highest-intensity muon beam with continuous time structure at Research Center of Nuclear Physics (RCNP) of Osaka University, Japan. A pion capture system using a superconducting solenoid magnet and a part of superconducting muon transport solenoid channel have been build in 2010. The highest muon production efficiency was demonstrated by the beam test carried out in February 2011. The result concludes that the MuSIC can provide more than $10^{9}$ muons/sec using a $400 \mathrm{~W}$ proton beam. The pion capture system is one of very important technologies for future muon programs such as moon to electron conversion searches, neutrino factories, and a muon collider. The MuSIC built the first pion capture system and demonstrate its potential to provide an intense muon beam. The construction on the entire beam channel of the MuSIC will be finished in five years. We plan to carry out not only an experiment to search the lepton flavor violating process but also other experiments for muon science and their applications using the intense muon beam at RCNP. The design performance has been confirmed by results of muon yield estimations from several beam tests.
\end{abstract}

36th International Conference on High Energy Physics

July 4-11, 2012

Melbourne, Australia

\footnotetext{
${ }^{1}$ Speaker, E-mail:y-hino@kuno-g.pnys.sci.osaka-u.ac.jp
} 


\section{The MuSIC system}

A high intense muon beam source, MuSIC, is now under construction at Research Center of Nuclear Physics (RCNP), Osaka University (Fig.1). MuSIC consists of a proton beam line, a pion production target with a pion capture solenoid, curved muon transport solenoids and a muon storage ring. It can achieve muon intensity of $10^{8}$ per second, using $392 \mathrm{MeV}$ and 1 micro A proton beam provided by the ring cyclotron, since the pion capture system improves the muon collection efficiency dramatically. Such a high intense muon beam will be used in various fields, for example, not only particle physics but also nuclear physics, chemistry, material science, accelerator or instruments $R \& D$ and so on. The pion capture solenoid system and transport solenoids with dipole field are most unique technologies in the MuSIC system.

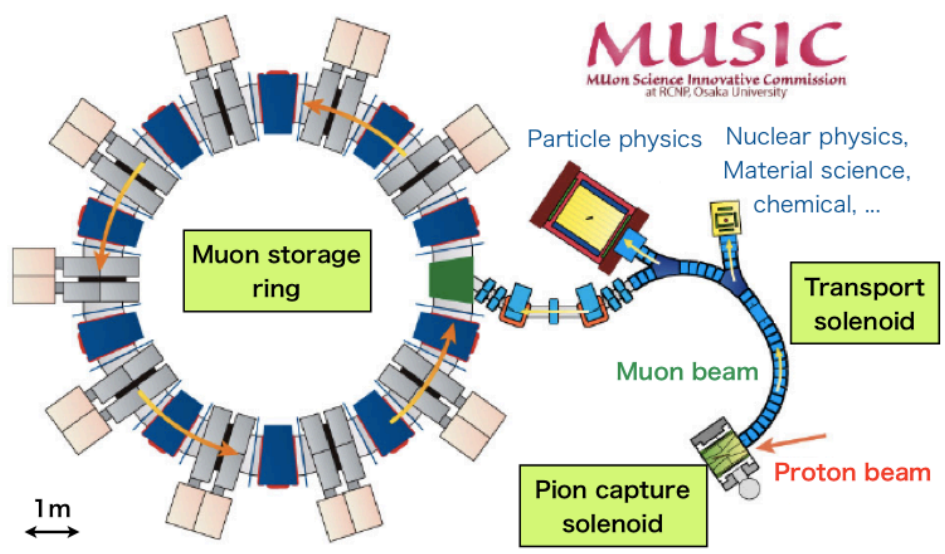

Fig. 1 Final layout of MuSIC

\subsection{Pion capture solenoid}

The pion and muon collection system is a key point for the highly efficient muon collection. In conventional muon facilities, there are strong limitation that proton beam loss at the pion production target must be small $(\sim 5 \%)$, because the neutron production target is located at downstream of the pion production target, so the thickness of the target must be enough thin, typical size is $2 \mathrm{~cm}$, and they cannot use magnetic field on the proton beam line, this limits solid angle of capture solenoid. On the other hand, in MuSIC, all protons from the ring cyclotron are used to produce pions and muons. The pion production target is very thick, $20 \mathrm{~cm}$ long and $40 \mathrm{~mm}$ in diameter, and it is located in a $3.5 \mathrm{~T}$ solenoidal magnetic field. So, generated pions after proton injection into the target can be collected with a large 
solid angle and transported. In short, MuSIC can achieve a high muon collection efficiency with thick production target and large capture solid angle, and these techniques would be used in future experiments of particle physics which need a very intense muon beam. For example, charged lepton flavor violation searches, COMET at J-PARC and Mu2e at Fermilab, and accelerator projects such as neutrino factories, muon colliders, and so on.

\subsection{Muon transport solenoids}

The captured pions decay into muons during passing the curved transport solenoids, whose maximum field is $2.0 \mathrm{~T}$. In this, charged particles move helically, and the center of the helical orbit moves to a direction perpendicular to the plane of the torus of the curved solenoid. With an additional dipole field to the same direction of the drift, the center position of the helical orbit is restored. The magnitude of the dipole field $B_{y}$ can be calculated by the following equation:

$$
B_{y}=\frac{p}{q r} \frac{1}{2}\left(\cos \theta+\frac{1}{\cos \theta}\right),
$$

where $p$ and $q$ refer the momentum and charge of the particle, $r$ is radius of the torus, and $\theta$ is an angle of particle direction.

Fig. 2 shows momentum distributions of muons with different $B_{y}$, simulated by g4beamline. We can select charge and momentum of the muon beam changing the dipole field.
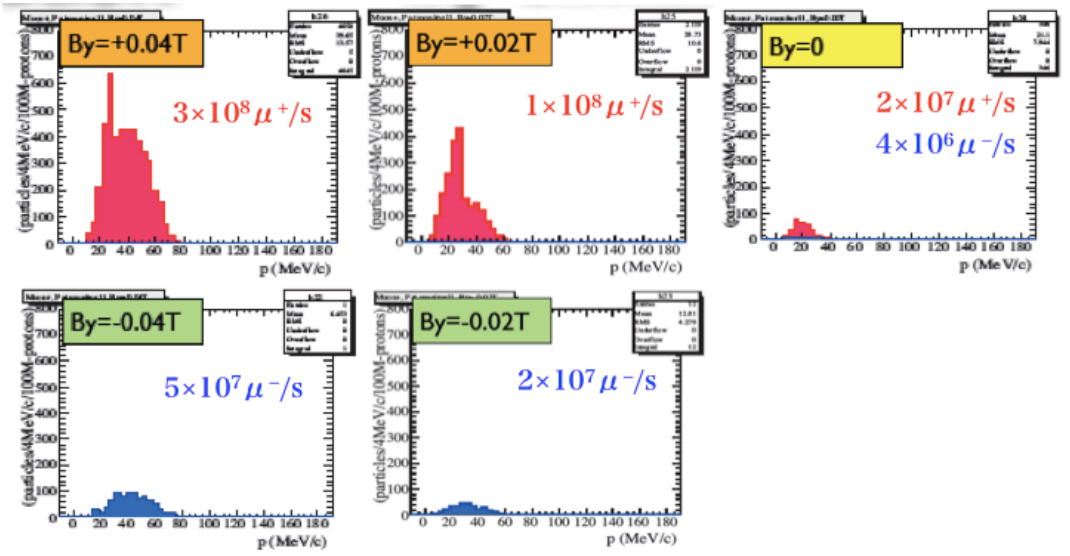

Fig. 2 Momentum distribution of muons at the transport solenoids 180 degrees exit

\subsection{Expected muon beam}

Using techniques above, MuSIC can provide very intense muon beam, which is same order with PSI or J-PARC MLF, but with small proton beam current. After 
comparison in Table, we can see that the muon collection efficiency (per beam power) of MuSIC is more than 1,000 times higher than that of the conventional facilities.

Currently, the muon transport solenoids are not completed, then the selection of muon charge or momentum is not enough, so beam is including lot of background particles (neutrons, pions, electrons, positrons, and so on) and it has wide spread of muon momentum.

Table. 1 Comparison of muon facilities in the world

\begin{tabular}{|c|c|c|c|}
\hline & MuSIC & PSI [1] & J-PARC [2] \\
\hline Beam power & $0.4 \mathrm{~kW}$ & $1200 \mathrm{~kW}$ & $1000 \mathrm{~kW}$ \\
\hline Muon intensity & $\sim 10^{8} / \mathrm{sec}$ & $10^{8} \sim 10^{9} / \mathrm{sec}$ & $\sim 10^{8} / \mathrm{sec}$ \\
\hline Time structure & continuous & continuous & pulsed $(25 \mathrm{~Hz})$ \\
\hline Beam polarization & medium & high & high \\
\hline Multiple use & only 1 channel & many channels & many channels \\
\hline
\end{tabular}

\subsection{Construction status}

The proton beam line, pion capture solenoid, and transport solenoids (36 degrees out of 180 degrees) have been constructed in 2009, and the construction of other parts will be done step by step, and it would finish probably in 2015 or 2016 .

\section{Beam tests at MuSIC}

In 2009, we finished the construction of present equipment of MuSIC, and from 2010 to now, we have carried out five beam tests with low proton beam current less than $\sim 500 \mathrm{pA}$ (because the radiation shield were not enough). To estimate the number of positive and negative muons separately, we measured muon lifetime and muonic X-rays. The experimental setup is shown in Fig.3. We used $\mathrm{Cu}$ and $\mathrm{Mg}$ metal as the muon-stopping target (Targ) and plastic scintillation counters as trigger counters (S1 and S2). Scintillators were read out by MPPCs (Multi-Pixel Photon Counter, Hamamatsu Photonics K.K.), which are not affected by magnetic field. To measure muonic X-rays, we placed Ge detector $(\mathrm{Ge})$ at the place beam does not hit and put shields to protect Ge from neutrons. 


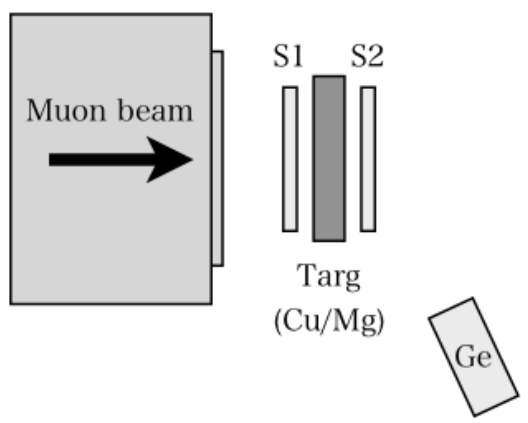

Fig. 3 Experimental setup

\subsection{Muon lifetime measurement}

In the material, the lifetime of negative muons $\tau_{-}$is smaller than that of positive muons $\tau_{+}$because of nuclear capture effect, so we can measure the yield of them respectively. But in case of $\mathrm{Cu}$ target, $\tau_{-} \sim 160 \mathrm{~ns}[3]$ while $\tau_{+} \sim 2.2 \mu \mathrm{s}$, the component of negative muons is small. So in this measurement, we mainly estimated the number of positive muons in the beam. Fig. 4 left shows the time spectrum of muon decay time.

\subsection{Muonic X-rays measurement}

When negative muon is captured into nuclear orbit and get down to the lower level, it emits characteristic X-rays, muonic X-rays, whose energy and intensity depends on the atomic number. Using energy spectrum (Fig.4 right) from data and Geant4 simulation, we estimated the negative muon yield.
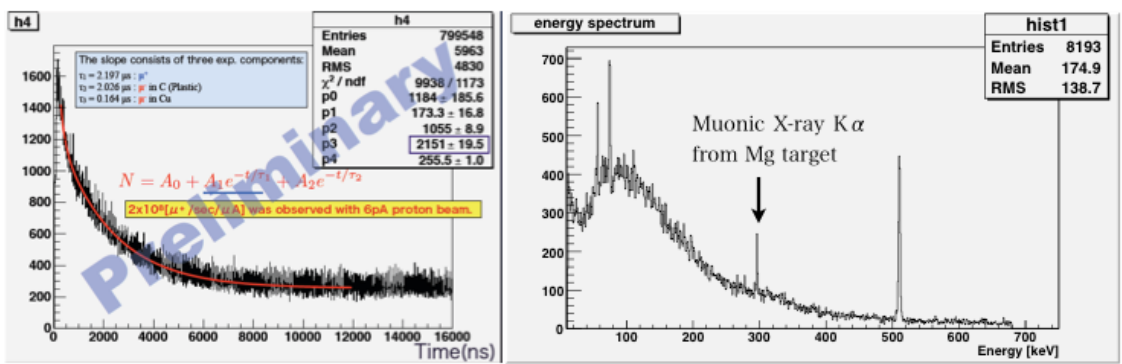

Fig. 4 Time spectrum of muon lifetime measurement (left) and energy spectrum of muonic $X$-ray measurement (right) 


\subsection{Comparison with simulation}

The results of muon yield estimations are summarized in Table 2. They showed good agreement with the simulation results, so we can conclude that MuSIC system works well as designed, in the point of number of muons.

Table. 2 Number of muons in the beam [/sec $/ 1$ micro A]

\begin{tabular}{|c|c|c|c|}
\hline & Measurement & Data & Simulation \\
\hline Positive muon yield & muon lifetime & $2 \times 10^{8}$ & $3 \times 10^{8}$ \\
\hline Negative muon yield & muonic X-ray & $(1.7 \pm 0.3) \times 10^{8}$ & $1.4 \times 10^{8}$ \\
\hline
\end{tabular}

\section{Summary}

MuSIC is the high intense DC muon source in Japan, which provides very high muon collection efficiency, $10^{8}$ muons per second, with $400 \mathrm{~W}$ proton beam. To achieve this intensity, we adopted new techniques to set thick pion production target inside the solenoid. By muon measurements at MuSIC, we confirmed that the present system works well and obtained muon yields are consistent with designed values.

\section{References}

[1] http://www.psi.ch/

[2] http://j-parc.jp/researcher/index-e.html

[3] T.Suzuki, D.F.Measday et.al., Physical Review C 35 (1987) 